\title{
Testing and Characterization of Different Papers as Substrate Material for Printed Electronics and Application in Humidity Sensor
}

\author{
Tijana Kojić, ${ }^{1}$ Goran M. Stojanović, ${ }^{1 *}$ Aleksandar Miletić, ${ }^{1}$ \\ Milan Radovanović, ${ }^{1}$ Hani Al-Salami, ${ }^{2}$ and Fabiana Arduini ${ }^{3}$ \\ ${ }^{1}$ University of Novi Sad, Faculty of Technical Sciences, Trg Dositeja Obradovića 6, 21000 Novi Sad, Serbia \\ ${ }^{2}$ Curtin Health Innovation Research Institute, Curtin University, 6102 Perth WA, Australia \\ ${ }^{3}$ Department of Chemical Science and Technologies, University of Rome "Tor Vergata", 00133 Rome, Italy
}

(Received July 12, 2019; accepted August 27, 2019)

Keywords: paper, printed and flexible electronics, characterization, humidity sensor

The use of papers as substrates in the process of manufacturing flexible electronic components is urgently required to obtain cost-effective products as well as to expand the potential applications of such components. This study aimed to examine the suitability of three different types of paper for sensor applications using an inkjet printing process. Three types of paper (denoted as Types 1,2, and 3) designed for specific applications in printed electronics were selected and entirely characterized in terms of microscopic and macroscopic properties, such as internal fibers structure, cross-sectional layer structure, surface roughness, and hardness. Dot arrays were printed on these three types of paper to determine how the papers absorb silver ink and which one is the best substrate for manufacturing printed electronic components. After a comprehensive analysis, the paper that exhibited the best feature was further studied as a substrate for printing interdigitated electrodes to develop a humidity sensor. The Type 2 paper-based sensor demonstrated the variation in capacitance in the range from 9.4 to $10.6 \mathrm{pF}$ while changing the relative humidity (RH) from 40 to $90 \%$. Thus, Type 2 has the great potential for application in flexible sensors, suggesting the possibility of industrial scalability and mass production of inexpensive, biodegradable, and conformable electronic components.

\section{Introduction}

There is a need for electronic devices with superior performance, robustness, smaller size, cost effectiveness, user friendliness, and lesser environmental impacts. ${ }^{(1)}$ Printed electronics is a rapidly emerging and innovative technology for the fabrication of electronic devices. Printed/flexible electronics enables the fabrication of electronic components on mechanically flexible substrates, such as plastic, paper, and textiles, using electrically functional inks and low-cost fabrication processes. ${ }^{(2)}$ The cost effectiveness, flexibility, and bendability 
of these substrates are attractive for high-performance foldable devices. ${ }^{(3)}$ When dealing with flexible devices, the substrate plays an important role in hosting various insulating, semiconducting, and conductive materials or components. Paper can be a good choice for a mechanically flexible substrate in printed electronics, because it is widely available and wellestablished in the printing and packing industries, it is biodegradable and lightweight, and it can be folded into different shapes and three-dimensional (3D) configurations. Furthermore, paper is a cost-effective and recyclable substrate compared with substrates made of plastic, such as polyethylene terephthalate (PET), since it is made of renewable raw materials. By employing the advantages of paper substrates, electronic circuits and sensors printed on paper are sustainable and capable of remaining functional, when folded at various angles and after repeated folding. Paper-based circuits and sensors can be easily trimmed by a pair of scissors and folded into complex shapes. ${ }^{(4)}$ Moreover, electronic components can be connected to both sides of a paper substrate, and paper substrates may be useful for applications in adhesive medical electrodes or low-cost portable diagnostic tools, exploiting the porosity of the paper. ${ }^{(5)}$ Paper of various compositions, thicknesses, surface roughnesses, weights, strengths, prices, and wetting properties is presently available. Different types of paper have been used as substrates for flexible electronic components, circuits, and electrochemical sensors. These include (a) Teslin paper (PPG Industry, USA), ${ }^{(4)}$ (b) pigment (kaolin)-coated paper (with a thickness of $130 \mu \mathrm{m}$ and a grammage of $\left.126 \mathrm{~g} / \mathrm{m}^{2}\right),{ }^{(6)}$ (c) glossy-finished photo paper (Epson C13S042187), ${ }^{(7)}$ (d) coated paper with a density of $65 \mathrm{~g} / \mathrm{m}^{2}$, a thickness of $76 \mu \mathrm{m}$, and a dielectric constant near 1.2 (Ahltrom manufacturer's data), ${ }^{(8)}$ (e) conventional printing paper from Gimba, SP, Brazil, ${ }^{(9)}$ Fabriano Copy $2\left(80 \mathrm{~g} / \mathrm{m}^{2}\right)$ and Fabriano Multip@per office paper $\left(100 \mathrm{~g} / \mathrm{m}^{2}\right),{ }^{(10)}$ and filter paper $\left(67 \mathrm{~g} / \mathrm{m}^{2}\right.$, Cordenons, Italy). ${ }^{(11)}$ In manufacturing processes, dry fabrication techniques, such as screen printing, inkjet printing, ${ }^{(8)}$ or a pen-on-paper approach, are usually employed. ${ }^{(12)}$ Different types of paper can also be used for biosensor manufacturing, and various fabrication methods from wax printing to origami-inspired approaches can be applied. ${ }^{(13)}$ A paperelectrode-based flexible pressure sensor modified with multiwalled carbon nanotubes (MWCNTs) was reported in Ref. 14. For applications in sensors, paper is a promising candidate owing to its porous structure and rough surface. ${ }^{(15)}$ The mechanical characterization of paper sheets was reported in Refs. 16 and 17, and a very good sensitivity towards changes in moisture and temperature was shown.

In the manufacturing of electronic components and sensors, the characteristics of the paper used impact the features of the final products. Although the producers of different types of paper provide limited information in data sheets such as the surface free energy of the front and reverse sides, weight, whiteness, temperature resistance, and printability, these data are not enough for researchers and professionals for electronic component development. The objectives of this study are to obtain detailed information about internal and surface characteristics of analyzed papers and relate them to the performance of the paper as a substrate for inkjet-printed sensor applications.

Herein, we give a complete characterization of three types of paper intended to be used as substrates in printed or flexible electronics. Three commercially available papers from Felix Schoeller Group ${ }^{(18)}$ were selected from the specific series p_e:smart ${ }^{\circledR}$ and denoted as Types 
1, 2, and 3. These types of paper were chosen because of their specific properties, such as (a) high dimensional stability regardless of temperature, (b) smooth surfaces, and (c) free from contamination. In detail, mechanical parameters, electrical parameters (capacitance), and printability by inkjet printing were analyzed to select the most suitable paper for manufacturing humidity sensors. Moreover, the sensor performance was comprehensively studied.

\section{Materials and Methods}

\subsection{Types of studied paper}

In this work, the three types of commercial paper from Felix Schoeller Group denoted as Types 1, 2, and 3 were studied. All three types have the same cross-sectional structure, consisting of raw paper in the middle, a resin coating below the raw paper, and a gloss coating (primer layer or nanoporous coating) on the top. Type 1 has a total thickness of around $115 \mu \mathrm{m}$ and a grammage of $190 \mathrm{~g} / \mathrm{m}^{2}$, Type 2 has a thickness of around $210 \mu \mathrm{m}$ and a grammage of $200 \mathrm{~g} / \mathrm{m}^{2}$, whereas Type 3 has a total thickness of $175 \mu \mathrm{m}$ and a grammage of $170 \mathrm{~g} / \mathrm{m}^{2}$. These types of paper were designed for printing electronic components as substrates, which have smooth surfaces and appropriate surface properties when silver ink is used for manufacturing components with high precision. The good interaction between the paper's coating and silver ink makes printing a sensor on a paper substrate reliable. The internal fiber structure of these types of paper provides high dimensional stability, which minimizes damage when constructing flexible printed sensors.

\subsection{Characterization techniques}

The following instruments were used for paper characterization. (1) For the analysis of morphological and topographic properties, scanning electron microscopy (SEM; EOL JSM 6460 LV) with energy dispersive X-ray spectroscopy (EDS) was used. (2) For surface roughness analysis, atomic force microscopy (AFM; Veeco di CP-II) a 3D optical profilometer, and a Huvitz microscope with Panasis software were used. (3) For mechanical characterization, a nanoindentation method was used with a G200 nanoindenter having a Berkovich diamond indenter with a face angle of $65.2^{\circ}$. (4) For humidity characterization, an Owlstone system was employed. This Owlstone system is an integrated test platform incorporating a humidity generator and a flow controller with exact measurements of temperature, flow, and humidity. The system can be used to produce a range of relative humidity $(\mathrm{RH})$ values from 40 up to $90 \% \mathrm{RH}$, with an accuracy of $\pm 1 \%$. (5) The contact angle and its hysteresis were measured by the sessile drop technique. A liquid droplet of about $2 \mu \mathrm{L}$ was dropped on the surface of the analyzed papers with a micropipette and the image of the droplet was captured by a digital camera (Tucsen camera, Xintu Photonics) attached to a microscope (Microzoom, Bausch, and Lomb) with a magnification of $2.25 \mathrm{x}$ and computed with the software IDS camera manager. The recorded droplet image was analyzed with the help of the DropSnake method implemented as a Java plug-in for the ImageJ software and based on B-spline snakes (active contours) to shape the drop. ${ }^{(19)}$ 


\section{Results and Discussion}

\subsection{Profilometer analysis}

To discover the internal structure of the three types of studied paper, profilometer analysis was conducted. The optical profiler was used to record 2D and 3D surface profiles of each sample, allowing the evaluation of the exact structure inside each type of paper, because it obtains a very large number of surface pictures from the bottom to the top and creates a single picture in two dimensions. The obtained 2D images are presented in Fig. 1, where Type 1 is magnified 20 times, Type 250 times, and Type 320 times.

From Fig. 1, it can be seen that cellulose fibers are the main constituent of each paper. The paper fibers have similar shapes and sizes, and there is little difference in the internal structure between the three types of paper. The internal structure of these types of paper demonstrated that they are suitable for the fabrication of electronic structures by inkjet printing because the fibrous structure is capable of absorbing applied functional inks. The good interaction between the paper's coatings and different inks can create reliable organic and printed electronics components.

\subsection{SEM results}

Structural characterization was performed by SEM to obtain exact cross-sectional views of the paper layers. SEM micrographs of the three types of studied paper with a magnification of around 500 times are presented in Fig. 2. From the SEM micrographs, the structures of the three types of paper with the gloss layer, raw paper, and resin coating (from left to right) are clearly visible, including their thicknesses.

\subsection{Surface characterization}

To determine the surface roughness of the studied paper samples, AFM was used, since it enables the determination of the surface topography of materials on the atomic scale vertically

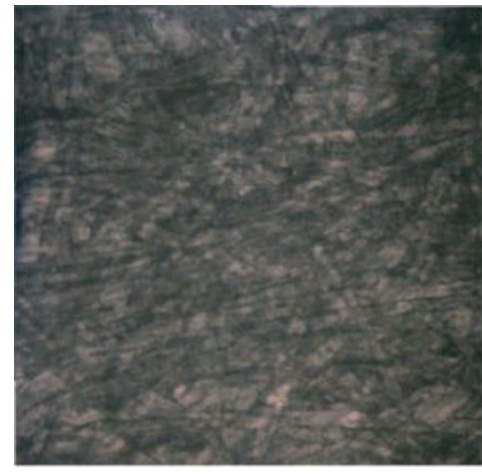

(a)

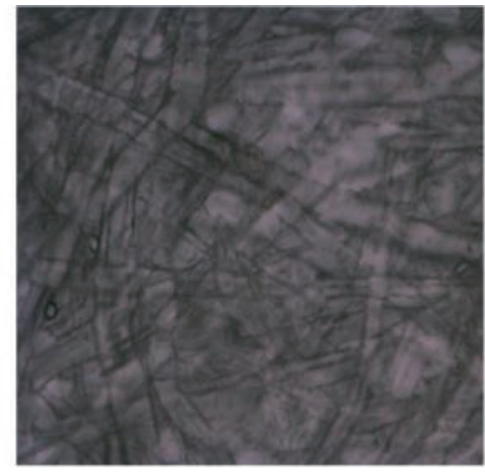

(b)

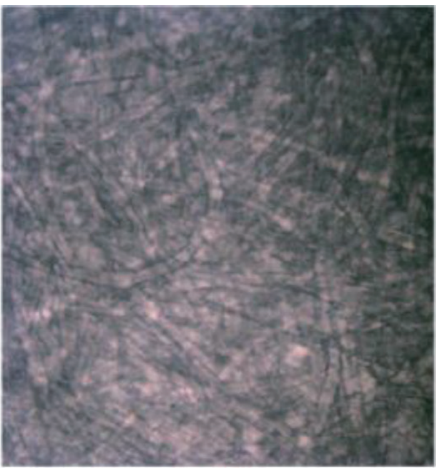

(c)

Fig. 1. (Color online) 2D profilometer images of studied papers: (a) Type 1, (b) Type 2, and (c) Type 3. 


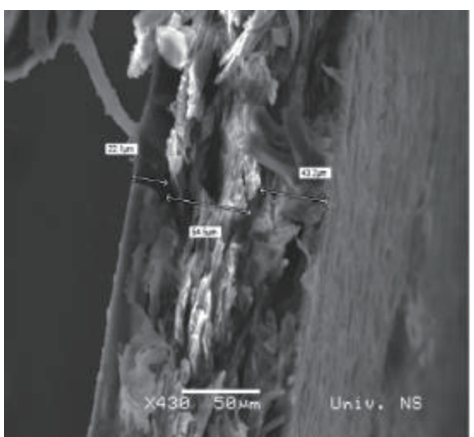

(a)

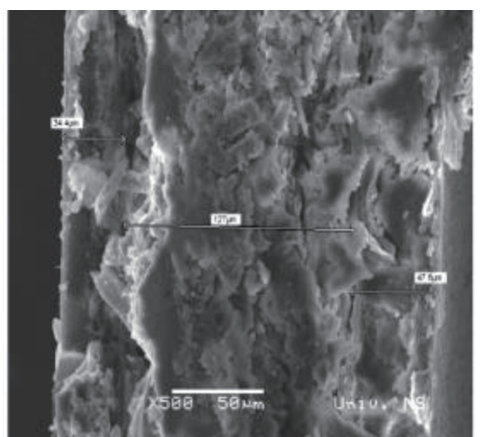

(b)

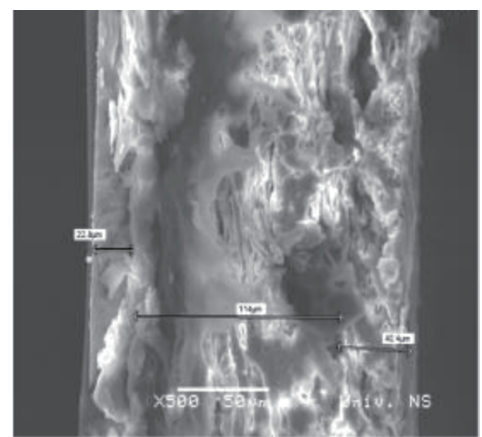

(c)

Fig. 2. SEM micrographs of papers: (a) Type 1, (b) Type 2, and (c) Type 3.

and on the nanometer scale horizontally. AFM images are presented in Fig. 3 for the three types of paper studied. Figures 3(a)-3(c) display the 3D surfaces and 2D images of Types 1, 2, and 3, respectively.

Surface roughness parameters for the three types of paper are presented in Table 1. They were determined as averages from four AFM images obtained for each paper sample. Parameters $S c i$ and $S v i$ show how much fluid might be stored in the core and valley zones. For a Gaussian height distribution, Sci approaches 1.56, while $S v i$ approaches 0.11. Large values of these indices indicate large void volumes in the core and valley zones. Since the indices of all three paper samples are close to those specific for a Gaussian height distribution, it might be considered that no sample has a special capability to retain fluid. $S d r$ indicates the ratio of the actual surface area to the projected area. For a totally flat surface, the actual surface area and projected area plane are the same and $S d r=0 \%$. When a surface is flat, the contact angle depends on the surface free energy. However, when a surface is rough, for the observed hydrophilic surfaces, the contact angle would be lower than the true contact angle. This is under the assumption that the whole surface area is wetted.

From the measured average surface roughness and $S d r$, one might expect the highest wetting for Type 1. However, wetting does not have to be complete and air pockets might be formed in surface cavities, which would reduce the wetting (increase the contact angle). The surface roughness of the paper as a substrate affects the line edge printing quality [see Fig. 11(b)]. The inkjet-printed conductive (Ag) line edge roughness decreases with decreasing surface roughness of the paper substrate.

\subsection{Mechanical characterization of studied paper}

The nanoindentation technique was employed to investigate the mechanical properties of small volumes of paper samples. The recorded load and depth of penetration were used to form the load-displacement curves that can be used to derive the hardness of the paper, because the paper can be exposed to various mechanical stresses or scratches during practical application. 

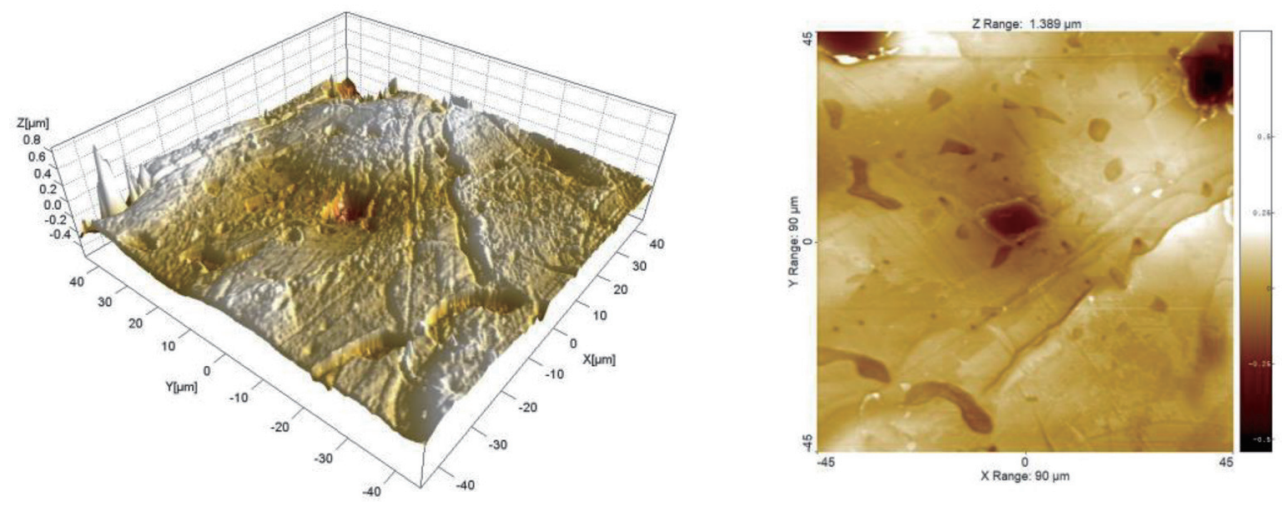

(a)
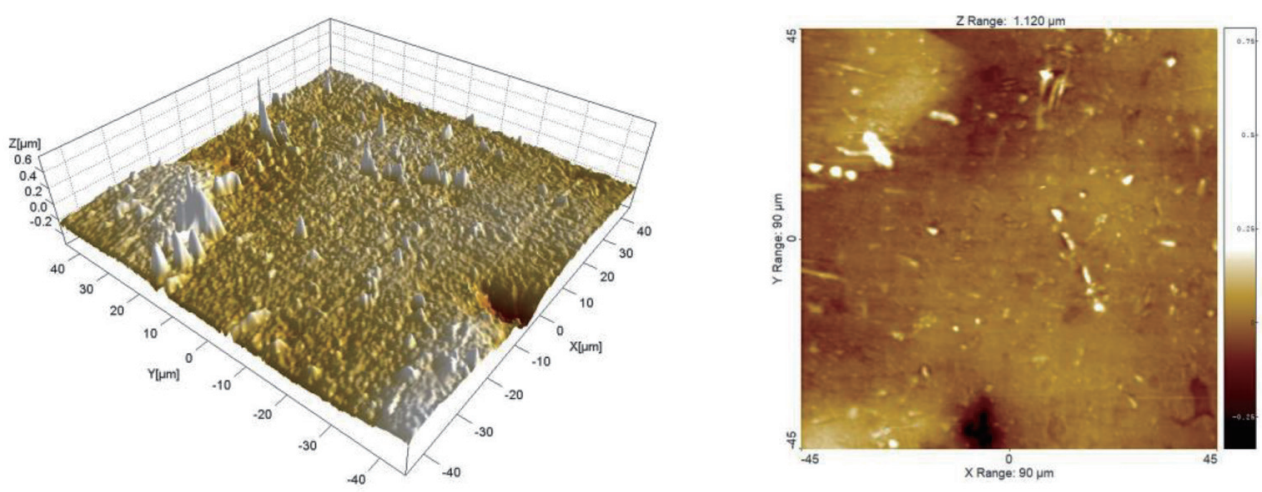

(b)
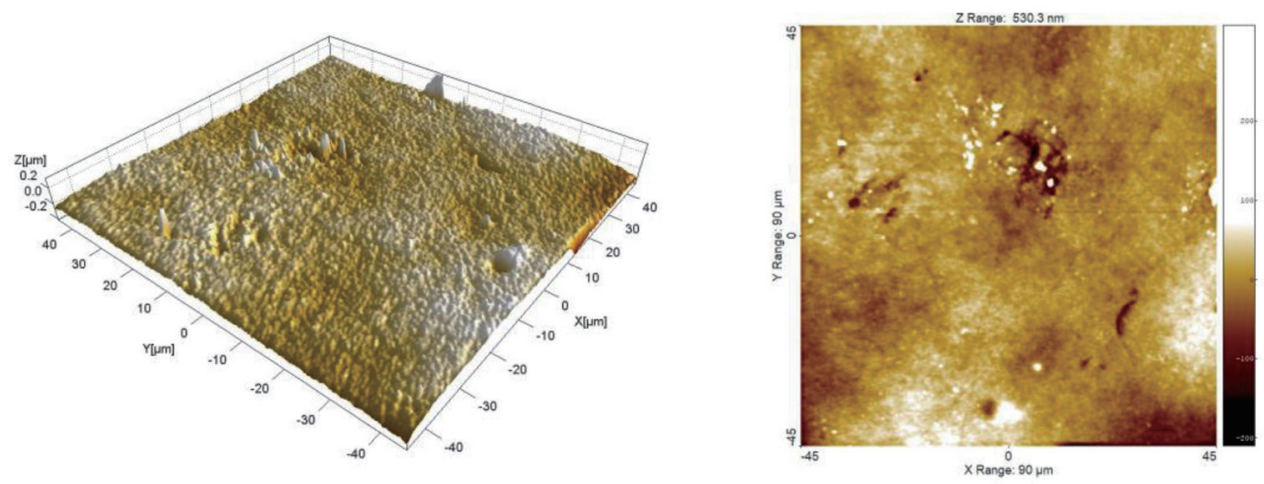

(c)

Fig. 3. (Color online) 3D and 2D AFM micrographs of papers: (a) Type 1, (b) Type 2, and (c) Type 3.

Table 1

Surface roughness parameters. $S a$, average surface roughness; $S q$, root mean square; $S c i$, core fluid retention index; $S v i$, valley fluid retention index; and $S d r$, surface area ratio.

\begin{tabular}{lccccc}
\hline & $S a(\mathrm{~nm})$ & $S q(\mathrm{~nm})$ & $S c i$ & $S v i$ & \multicolumn{1}{c}{$S d r(\%)$} \\
\hline Type 1 & $68 \pm 22$ & $102 \pm 35$ & $1.27 \pm 0.14$ & $0.12 \pm 0.03$ & $1.297 \pm 1.36$ \\
Type 2 & $35 \pm 6$ & $50 \pm 6$ & $1.43 \pm 0.14$ & $0.12 \pm 0.01$ & $0.21 \pm 0.02$ \\
Type 3 & $25 \pm 3$ & $34 \pm 3$ & $1.5 \pm 0.09$ & $0.12 \pm 0.02$ & $0.12 \pm 0.02$ \\
\hline
\end{tabular}


Nanoindentation measurements for different sets of samples were carried out using a G200 nanoindenter equipped with a Berkovich triangular pyramidal diamond tip. A preset depth (maximum of $5 \mu \mathrm{m}$ ) was applied to the pyramidal indenter in contact with the paper surface. The indentation cycle was considered as a load-unload cycle; first, a load was applied, ramped up to a peak, and then maintained for a fixed time before unloading. The time to ramp up the load was set to $15 \mathrm{~s}$, while the peak loading time was set to $10 \mathrm{~s}$. Multiple nanoindentation tests were carried out with at least eight indentations to ensure the repeatability of the measured results. Figure 4 shows the mean load-displacement curves for Types 1, 2, and 3.

Figure 4 shows that in the loading process at the maximum preset depth of $5 \mu \mathrm{m}$, the penetration loads are around $15 \mathrm{mN}$ for Type $1,9 \mathrm{mN}$ for Type 3 , and $2 \mathrm{mN}$ for Type 2 . Note from Fig. 4 that the displacement into the surface exceeds the preset depth of $5 \mu \mathrm{m}$ by approximately $500 \mathrm{~nm}$. This happens when the Berkovich tip reaches the given preset depth and is held there for $10 \mathrm{~s}$; as set under the initial conditions, the tip enters the material to a depth of a few more nanometers. It was confirmed that the raw paper layer was not reached (the gloss layer thicknesses are $34 \mu \mathrm{m}$ for Type 2 and around $22 \mu \mathrm{m}$ for Types 1 and 3, as shown in Fig. 2). The indentation curves for Types 1 and 2 show an increase in indenter depth (secondary penetration) during the plateaus while maintaining a constant load and after loading; they also show a decrease in load at the end of the unloading curve. In almost all the curves, the viscoelastic return of the samples was observed. It can be concluded from Fig. 4 that Type 2 has the softest gloss layer because it needed the smallest load to reach the preset depth of $5 \mu \mathrm{m}$. Comparing the results of the other two types, both types have gloss coatings with similar thicknesses, but Type 3 has a raw paper layer with a thickness that is almost twice that of Type 1 . Therefore, a smaller load is needed to reach the same preset penetration depth of $5 \mu \mathrm{m}$ for Type 3. Figure 5 shows nanoindentation marks after evaluating the mechanical properties. They are triangular pyramidal shapes on the paper substrate and their dimensions are also depicted in Fig. 5. The porous surface structure of the paper can be distinctly observed in Fig. 5, which is advantageous for fabrication by inkjet printing.

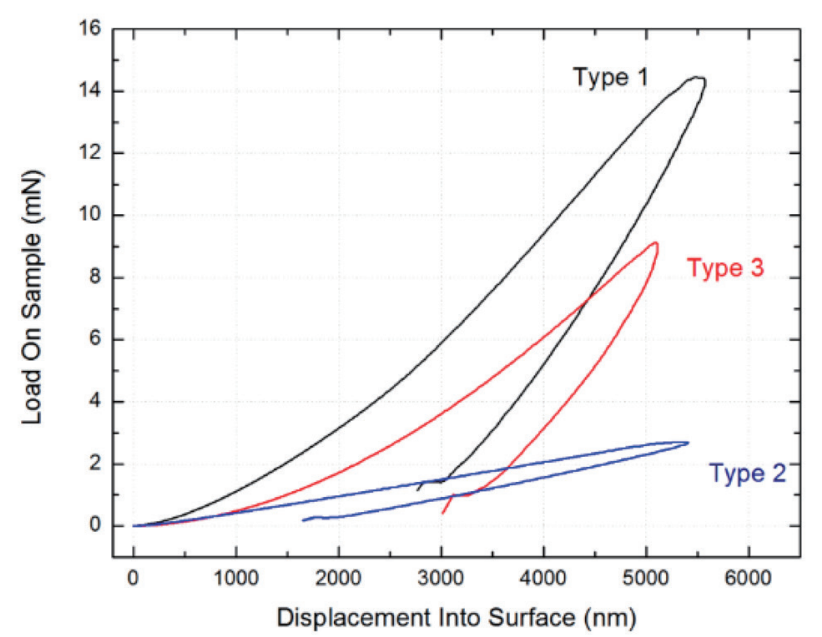

Fig. 4. (Color online) Load-displacement curves for analyzed papers. 


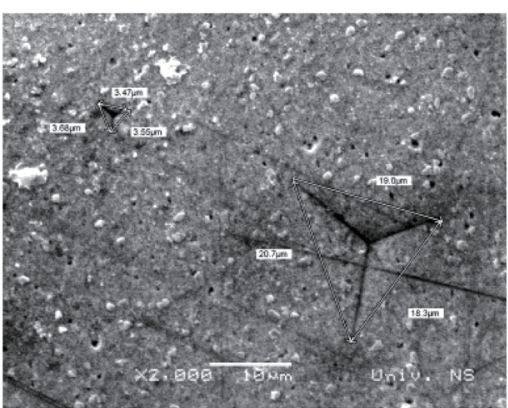

(a)

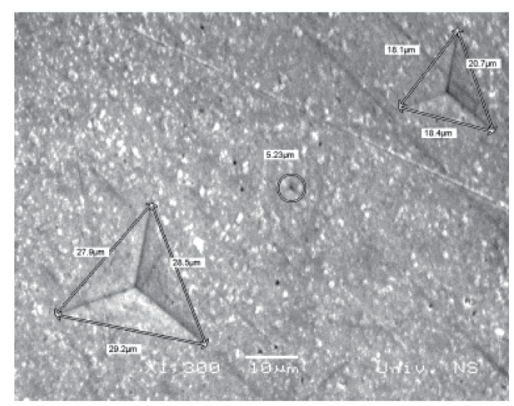

(b)

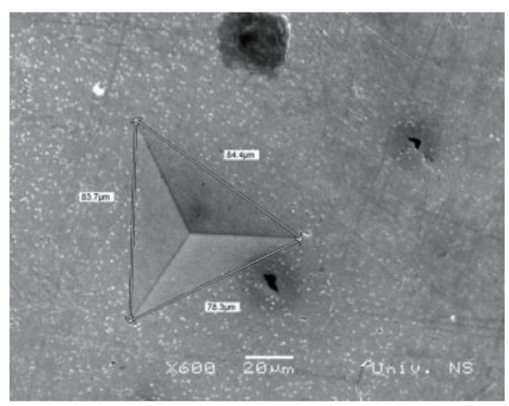

(c)

Fig. 5. Marks after nanoindentation testing: (a) Type 1, (b) Type 2, and (c) Type 3.

\subsection{Wettability of paper}

Wetting properties are very important when an inkjet printing process is applied. Thus, we performed a drop-shape analysis to measure static contact angles from the optical images saved and analyzed with the software tool ImageJ (option drop analysis). The variation in static contact angle as a function of time was measured (at room temperature and a humidity of around 60\%) and results are presented in Fig. 6(a) for water droplets and in Fig. 6(b) for silver ink droplets. The contact angles obtained for the three types of studied paper were below $90^{\circ}$, which corresponds to high wettability. Type 2 had the highest contact angle, followed by Types 1 and 3. This proves that Type 2 is the best substrate in the inkjet process owing to its absorptive surface suitable for silver ink and can be used at high temperatures. Type 2 had a medium surface roughness, ensuring that the highest measured contact angle. Types 1 and 3 showed low contact angles, which indicates that ink can be spread over a large are on their surfaces and controlling the exact geometrical shapes to be fabricated is not trivial. The obtained contact angles (Fig. 6) are slightly higher than those reported in Ref. 20 for the same silver ink on polyimide (PI) film and silicon wafer substrates, whose measured values were 10.6 and $16.3^{\circ}$, respectively. Furthermore, our measured results are comparable to those presented in Ref. 21 for water (in the range from 8.079 to $36.412^{\circ}$ ) and Ag nanoparticle ink (in the range from 8.293 to $19.949^{\circ}$ ) on different substrates (glass, silicon, PET, ITO, and PVA). The inkjet printing line width can be larger if the contact angle of the droplet with the substrate is higher. Thus, it can be concluded that the printing line width is affected by the contact angle rather than by the surface roughness of the substrate.

Wettability is not just a static phenomenon. For nonhomogeneous paper surfaces, there are domains or surfaces that prevent smooth drop motion. Thus, we also measured the contact angle hysteresis, which is depicted in Fig. 7, for the three types of paper studied. The observed behavior is similar to that of the measured static angle.

Furthermore, the surface tension of the three types of paper was tested ${ }^{(18)}$ and the measured values on the front side of the paper were as follows: Type $1=55 \pm 10 \mathrm{mN} / \mathrm{m}$, Type $2=45 \pm$ $10 \mathrm{mN} / \mathrm{m}$, and Type $3=65 \pm 10 \mathrm{mN} / \mathrm{m}$. The strongest wetting was observed in Type 3 , which showed the highest surface tension, and the weakest wetting was observed in Type 2, which 


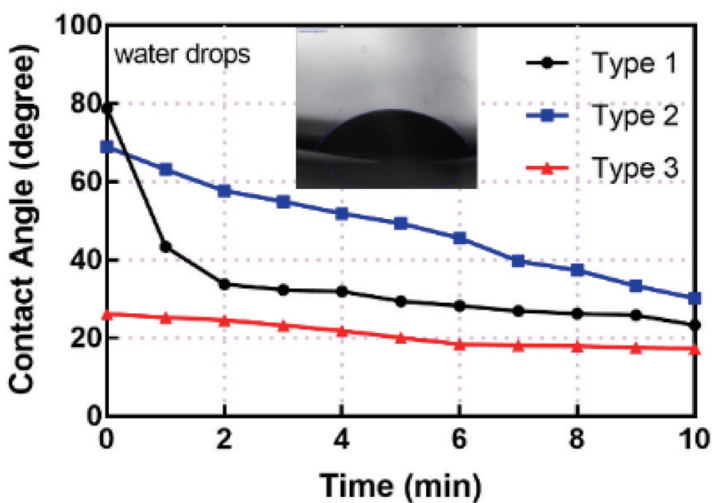

(a)

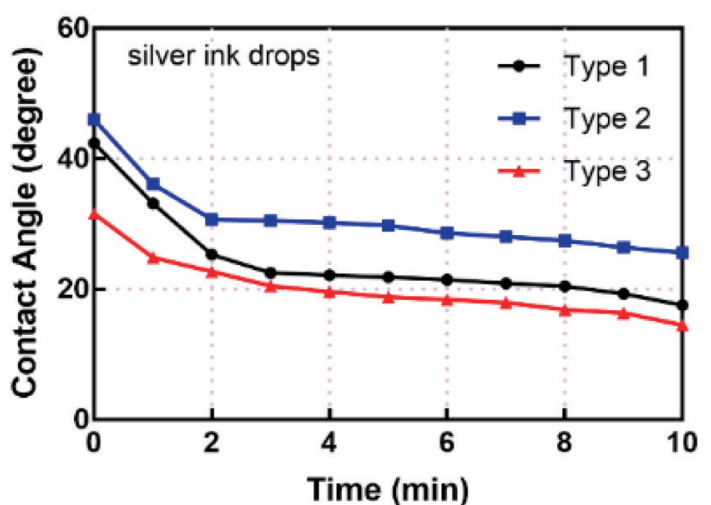

(b)

Fig. 6. (Color online) Contact angle as a function of time (drop age) for (a) water drops and (b) silver ink drops, on the three types of paper studied.

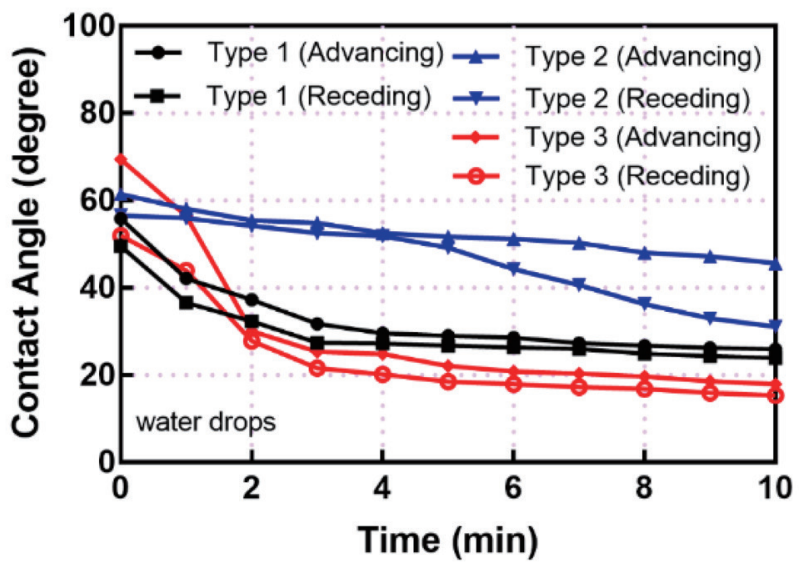

Fig. 7. (Color online) Contact angle hystereses for three types of paper studied.

showed the lowest surface free energy. The results presented in Fig. 6 are in good agreement with these surface tension results. The higher the surface free energy, the stronger the wetting and the lower the contact angle. The high surface tension of paper substrates indicates that the conductive line is not perfectly continual and that the silver ink spreads uncontrollably over the surface during inkjet printing.

\subsection{Inkjet-printed dot arrays on different types of paper}

Because of the intended application of the analyzed paper in printed and flexible electronics, dot arrays were printed on all three types of paper to determine how each paper absorbs silver ink and which is the best substrate for flexible electronics. All dots were formed by inkjet printing using a Dimatix DMP3000 deposition material printer. ${ }^{(22)}$ Drops were printed on 2 
$\times 2 \mathrm{~mm}^{2}$ pieces of paper for all three types of analyzed samples to observe how each paper absorbs the applied functional ink. Before printing, the stand (holder) on which the paper was placed was heated to $40{ }^{\circ} \mathrm{C}$ so that the silver ink did not spill out from the printing line on the gloss layer of the paper. Commercially available $50 \mathrm{wt} \%$ Sigma-Aldrich silver nanoparticle ink dispersed in tripropylene glycol monomethyl ether ${ }^{(23)}$ was used to print dot arrays. Printed silver dots on the three types of studied paper are presented in Fig. 8. The distance between the centers of adjacent dots was $34 \mu \mathrm{m}$. The diameter of one drop of silver ink on the substrate was around $20 \mu \mathrm{m}$.

As shown in Fig. 8(a), the silver ink has a poor reaction with Type 1 and the light color of the dots indicates that sintering has not been fully completed. This is due to the fact that Type 1 has no nanoporous coating on the top. From Fig. 8(c), it can be seen that the drops on Type 3 paper are not circular because of the highest surface tension and also the extremely smooth surface of this paper [Fig. 3(c)], which may induce the drop spillage effect. Type 2 has the best reaction with the silver ink and the drops are circular, as can be seen in Fig. 8(b). The thicknesses of the layers and their ratio (gloss layer to raw paper) affect the absorption capacity of the silver ink during the inkjet printing. For the Sigma-Aldrich silver nanoparticle ink used for the fabrication, the best choice is Type 2. The ink was seamlessly absorbed and uniform printing was achieved. The thinner gloss layer of Type 1 causes the ink to be mostly retained in the top gloss layer, which results in faster sintering during the heating of the holder, while the raw paper, being thicker than the gloss layer of Type 3, absorbs too much ink, and no wellformed dots are produced during the printing. Thus, it can be concluded that the best paper for future application in the field of printed and flexible electronics is Type 2.

\subsection{Humidity sensor on paper substrate}

Paper is principally made of cellulose fibers, which possess hygroscopic properties. This varies the electrical components in a circuit and degrades metal deposits and the substrate itself. The experimental setup and equipment for measurement are depicted in Fig. 9. A humidity generator was used for ensuring adequate humidity in the range from 40 to $90 \%$ in the test chamber (a glass bottle), regulating the input and output of wet airflow. The fabricated sensor (Fig. 11) was placed in this test chamber and two wires were connected with two electrodes of the

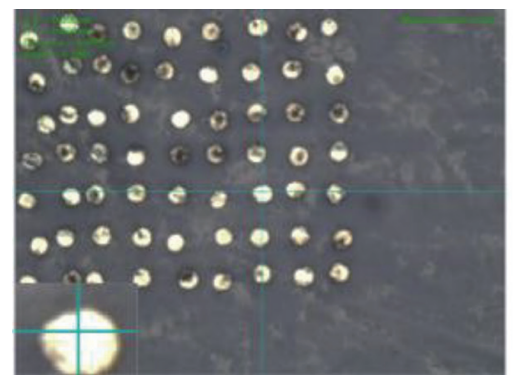

(a)

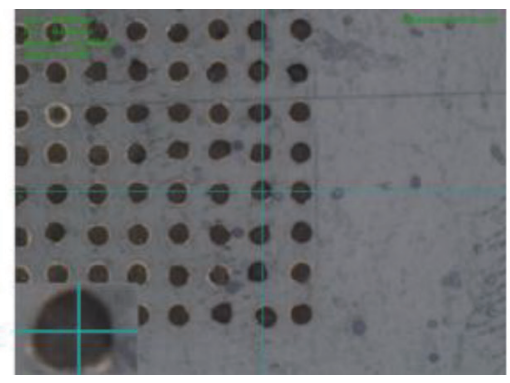

(b)

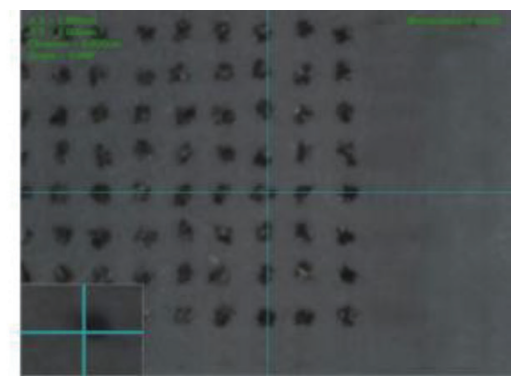

(c)

Fig. 8. (Color online) Dot arrays inkjet-printed on different types of paper: (a) Type 1, (b) Type 2, and (c) Type 3. 


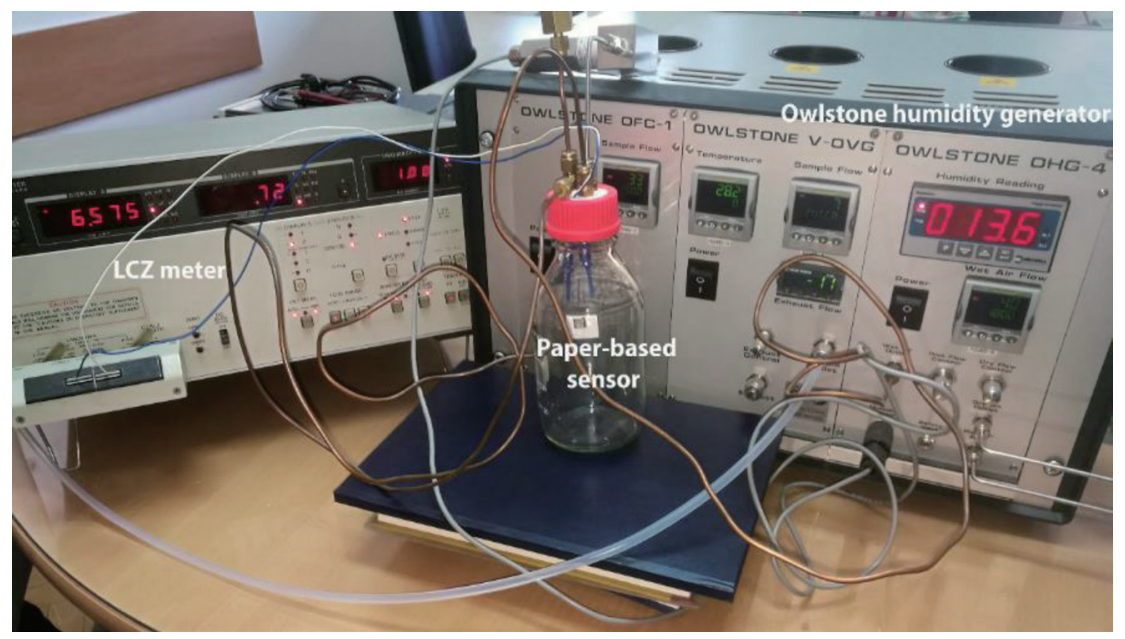

Fig. 9. (Color online) Experimental setup for testing humidity sensor.

interdigitated capacitor from one side. From another side, these two wires were attached to the sample holder of the LCZ meter HP4277A. The measurement was carried out under laboratory conditions, and the temperature was in the range from 23.2 to $23.6^{\circ} \mathrm{C}$.

To measure the humidity, an interdigitated capacitive (IDC) electrode structure was printed by inkjet printing, and afterwards sintered at $100{ }^{\circ} \mathrm{C}$ for $30 \mathrm{~min}$, only on Type 2 paper, because it showed the best results after dot array printing with the silver nanoparticle ink, as demonstrated in the previous subsection. Figure 10 shows the design of the IDC electrode structure composed of nine electrodes with their corresponding dimensions. The overall dimension of the capacitor was $8.6 \times 5.9 \mathrm{~mm}^{2}$ and the length of each electrode was $5 \mathrm{~mm}$, while the width and gap were 0.3 and $0.4 \mathrm{~mm}$, respectively.

Figure 11(a) displays the interdigitated structure printed on Type 2 paper, whereas Fig. 11(b) presents the conductive line edge of the printed structure on this paper. It can be observed that the printing layer is uniform and that the edge is formed without spraying silver ink drops over the surrounding area. The surface roughness of the paper as a substrate affects the line edge printing quality [Fig. 11(b)]. The inkjet-printed conductive (Ag) line edge roughness decreases with decreasing surface roughness of the paper substrate.

In the case of an interdigitated capacitor, the electric field lines are closed also through the substrate, which is paper in our case, and the other part of electrical field is closed through air, which represents the dielectric between the fingers of the interdigitated capacitor. With increasing air humidity, the airflow rate increases, which directly increases the capacitance of the interdigitated capacitor. With decreasing air humidity, the permittivity decreases, which also reduces the capacitance of the IDC electrode structure.

Figure 12 depicts the variation in sensor capacitance as a function of $\mathrm{RH}$ in the range from 40 to $90 \%$, with increasing and decreasing humidity. The IDC paper-based sensor demonstrated a slight hysteresis between the up and down cycles. The reason for this phenomenon is that the surface of the paper reacts with moisture. When it is exposed to different humidity levels, the 


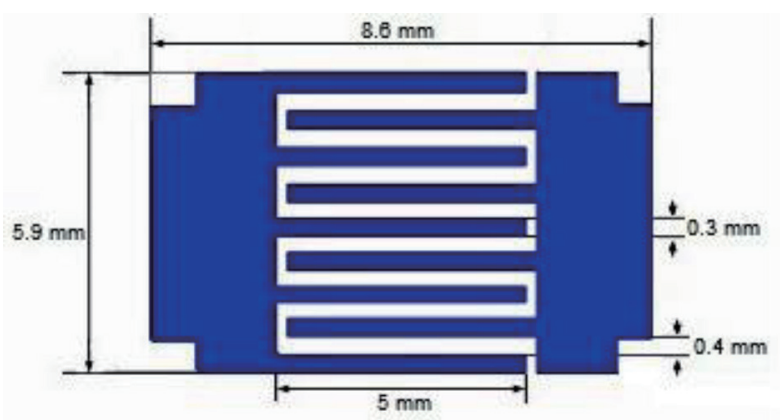

Fig. 10. (Color online) Designed IDC electrode structure with corresponding dimensions.

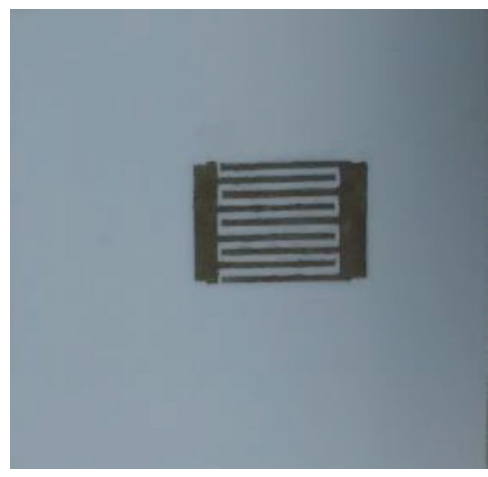

(a)

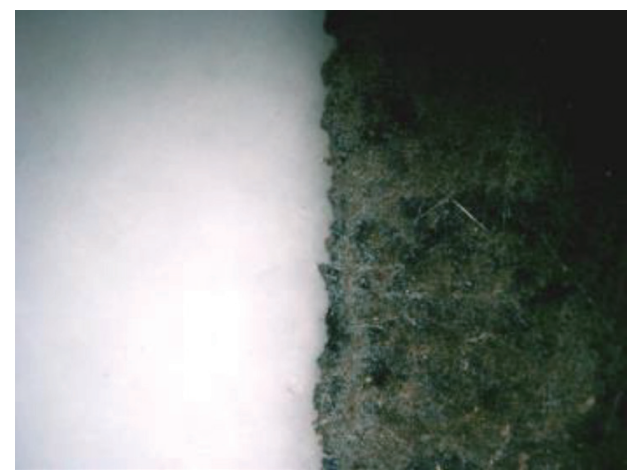

(b)

Fig. 11. (Color online) IDC electrode structure manufactured on Type 2 paper by inkjet method. (a) Appearance after printing and (b) enlargement of the conductive line edge of the printed component.

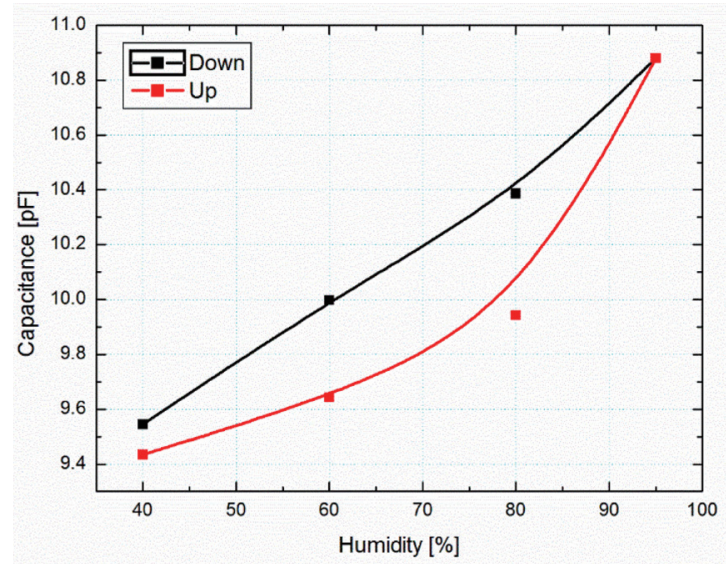

Fig. 12. (Color online) Behavior of inkjet-printed sensor in an Up-Down cycle in the range of 40-90\% RHs. The Up (in red) and Down (in black) curves correspond to the adsorption and desorption processes, respectively.

printed sensor adsorbs and desorbs different amounts of water from the surrounding ambient. If $\mathrm{RH}$ is high, the sensor will absorb more water vapor. If RH is reduced to the initial low level of $40 \%$, it will take more time for the sensor to desorb water and reach equilibrium. When using paper as a substrate, the adsorption process is relatively fast, whereas the desorption process 


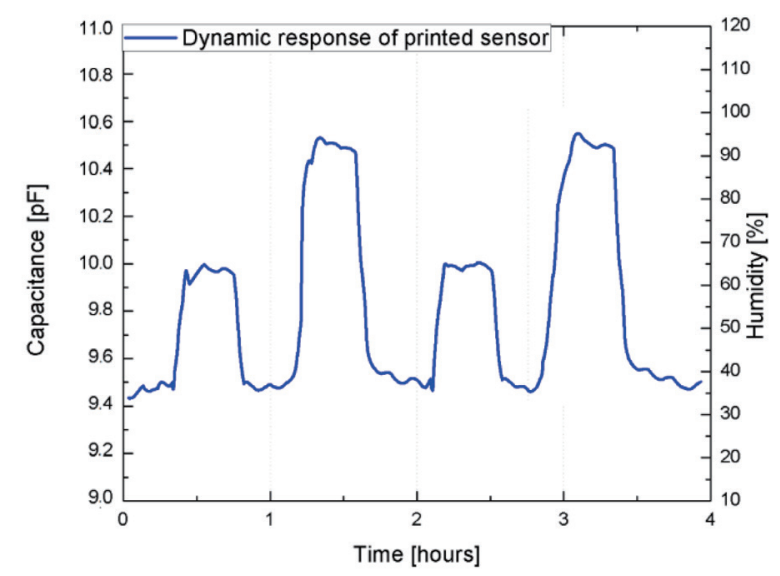

Fig. 13. (Color online) Dynamic response of a printed IDC sensor at a frequency of $100 \mathrm{kHz}$.

until equilibrium is reached is slow owing to the cellulose fibers in the internal structure of the paper. These cycles showed an increase in RH (Up) and a decrease in RH (Down) for the single measurement cycle at $100 \mathrm{kHz}$ (shown in Fig. 12). The nonlinear fitting curves are also displayed. The water molecules would accumulate and interacting cohesion forces would increase exponentially after reaching a threshold, as evidenced by water activity versus sorption isotherm. Changes in the water profile affect the permittivity, consequently causing the capacitance to change. The cohesion and adhesion of the water molecules depend on the structure of Type 2 paper, as shown in SEM and AFM micrographs. The hysteresis can be reduced by using thinner paper, because it will take less time for the paper structure to absorb water molecules and reach the equilibrium state.

The reproducibility of the results of the inkjet-printed sensor was tested at $20 \mathrm{~min}$ intervals with RH being increased and decreased stepwise in the range from 40 to $90 \%$. A period of 20 min was set to allow the diffusing water molecules to be adsorbed between the electrodes (fingers) of the IDC structure and to stabilize RH. The reproducibility of the results of the sensor exposed to different $\mathrm{RH}$ values over a period of about $4 \mathrm{~h}$ at room temperature is shown in Fig. 13.

The dynamic response of the printed sensor shows the repeatability of the results during a period in which RH changes over time. After 20 min of holding RH at $40 \%$ for which the capacitance of the printed sensor is $9.45 \mathrm{pF}$, about $5 \mathrm{~min}$ is required for the sensor to respond to a higher RH. The printed sensor has a capacitance of about $10 \mathrm{pF}$ at a higher RH of $60 \%$. After that, RH is returned to $40 \%$ for 20 min, during which the sensor returns to the previous capacitance, then $\mathrm{RH}$ is increased to $90 \%$, at which the capacitance is about $10.6 \mathrm{pF}$.

\section{Conclusions}

The use of paper in microelectronics and flexible electronics is currently in its infancy. In this work, we comprehensively studied the internal structures of fibers of three types of paper (using an optical profilometer) and their surface roughness characteristics (by AFM), cross- 
sectional structure (by SEM), and hardness (using a nanoindenter). Among the three types of paper studied as substrates, the most promising paper type for the inkjet-printed sensor is Type 2 . This paper has a precoating and a nanaporous coating (both parts of a gloss layer) at the top of its cross section. This work proved that surface roughness should be neither very large (Type 1) nor extremely small (Type 3). This condition is satisfied by Type 2. Type 2 has the lowest surface tension, ensuring the excellent quality of inkjet-printed lines as well as the lowest mechanical hardness, which is also a promising property for realizing a sensor system that is flexible in its entirety so as to be conformable to a desired shape. The incorporation of suitable types of paper in the inkjet-printing process is innovative, economical, and efficient, and has great potential for various applications. The most suitable paper was selected for the fabrication of a humidity sensor, and graphs of capacitance as a function of humidity and capacitance as a function of time were provided. The humidity-detection features of paper can play a significant role in the suitability and quality of the printing process and final product. Accordingly, the application of paper to inexpensive sensors have great promise at the industrial scale, but comprehensive characterization and accurate profiling of the features of paper are required, as performed and described in this study.

\section{Acknowledgments}

This study received funding from the EU Horizon 2020 research and innovation programme under the MSC Grant agreement no. 690876-MEDLEM and was partly supported by the project 142-451-2459/2018-03. The authors would like to thank Schoeller Technocell GmbH \& Co., KG for valuable comments. They are also grateful to their colleagues (G. Dubourg) in the Biosens Institute, UNS, for assistance in measuring contact angles.

\section{References}

1 C. Yang, H. Gu, W. Lin, M. M. Yuen, C. P. Wong, M. Xiong, and B. Gao: Adv. Mater. 23 (2011) 3052. https:// doi.org/10.1002/adma.201100530

2 C. Paragua, K. Frigui, S. Bila, and D. Baillargeat: Proc. 45th European Microwave Conf. (2015). https://doi. org/10.1109/EuMC.2015.7345900

3 Q. Cheng, Z. Song, T. Ma, B. B. Smith, R. Tang, H. Yu, H. Jiang, and C. K. Chan: Nano Lett. 13 (2013) 4969. https://doi.org/10.1021/n14030374

4 Y. Wang, H. Guo, J. J. Chen, E. Sowade, Y. Wang, K. Liang, K. Marcus, R. R. Baumann, and Z. Sh. Feng: ACS Appl. Mater. Interfaces 8 (2016) 26112. https://doi.org/10.1021/acsami.6b06704

5 A. C. Siegel, S. T. Phillips, M. D. Dickey, N. Lu, Z. Suo, and G. M. Whitesides: Adv. Funct. Mater. 20 (2010) 28. https://doi.org/10.1002/adfm.200901363

6 A. Määttänen, P. Ihalainen, P. Pulkkinen, S. Wang, H. Tenhu, and J. Peltonen: ACS Appl. Mater. Interfaces 4 (2012) 955. https://doi.org/10.1021/am201609w

7 O. S. Kwan, H. Kim, H. Ko, J. Lee, B. Lee, C. H. Jung, J. H. Choi, and K. Shin: Carbon 58 (2013) 116. https:// doi.org/10.1016/j.carbon.2013.02.039

8 M. Balde, F. Jacquemoud-Collet, and A. V. B. Sorli: Sens. Actuators, A 240 (2016) 118. https://doi.org/10.1016/ j.sna.2015.09.037

9 M. Santhiago, C. C. Corrêa, J. S. Bernardes, M. P. Pereira, L. J. M. Oliveira, M. Strauss, and C. C. B. Bufon: ACS Appl. Mater. Interfaces 9 (2017) 24365. https://doi.org/10.1021/acsami.7b06598

10 S. Cinti, M. Basso, D. Moscone, and F. Arduini: Anal. Chim. Acta 960 (2017) 123. https://doi.org/10.1016/ j.aca.2017.01.010

11 S. Cinti, C. Minotti, D. Moscone, G. Palleschi, and F. Arduini: Biosens. Bioelectron. 93 (2017) 46. https://doi. org/10.1016/j.bios.2016.10.091 
12 A. Russo, B. Y. Ahn, J. J. Adams, E. B. Duoss, J. T. Bernhard, and J. A. Lewis: Adv. Mater. 23 (2011) 3426. https://doi.org/10.1002/adma.201101328

13 A. T. Singh, D. Lantigua, A. Meka, S. Taing, M. Pandher, and G. Camci-Unal: Sensors 18 (2018) 2838. https:// doi.org/10.3390/s18092838

14 Z. Yu, Y. Tang, G. Cai, R. Ren, and D. Tang: Anal. Chem. 91 (2019) 1222. https://doi.org/10.1021/acs. analchem. 8 b04635

15 J. Sarfraz, P. Ihalainen, A. Maatanen, and J. Peltonen: Thin Solid Films 534 (2013) 621. https://doi.org/10.1016/ j.tsf.2013.02.055

16 E. Linvill, P. Larsson, and S. Ostlund: Mater. Des. 128 (2017) 231. https://doi.org/10.1016/j.matdes.2017.05.002

17 E. Linvill and S. Ostlund: Exp. Mech. 64 (2014) 1329. https://doi.org/10.1007/s11340-014-9898-7

18 Special papers for printed electronics: https://www.felix-schoeller.com/en_en/business-unit/other-specialtypapers/p-esmartr.html (accessed July 2019).

19 A. F. Stalder, G. Kulik, D. Sage, L. Barbieri, and P. Hoffmann: Colloids Surf. A 286 (2006) 92.

20 M. Y. Chuang: Protoc. Rep. Pap. 51 (2018) https://repository.upenn.edu/scn_protocols/51 (accessed August 2019).

21 V. K. S. Yadav, G. Natu, and R. Paily: IEEE Trans. Compon. Packag. Manuf. Technol. 8 (2018) 1678. https:// doi.org/10.1109/tcpmt.2018.2854629

22 DMP-3000 Materials Printer: https://www.fujifilmusa.com/press/news/display_news?newsID=880182 (accessed July 2019).

23 Silver nanoparticle ink: https://www.sigmaaldrich.com/catalog/substance/ silvernanoparticleink1078798765?lang=en\&region=SX (accessed July 2019). 